

\author{
INEEL/CON-04-01593 \\ PREPRINT
}

\title{
Verification And Validation Of The Tritium Transport Code TMAP7
}

\author{
Glen R. Longhurst \\ James Ambrosek
}

September 12, 2004

\section{$7^{\text {th }}$ International Conference on Tritium Science and Technology}

This is a preprint of a paper intended for publication in a journal or proceedings. Since changes may be made before publication, this preprint should not be cited or reproduced without permission of the author.

This document was prepared as an account of work sponsored by an agency of the United States Government. Neither the United States Government nor any agency thereof, or any of their employees, makes any warranty, expressed or implied, or assumes any legal liability or responsibility for any third party's use, or the results of such use, of any information, apparatus, product or process disclosed in this report, or represents that its use by such third party would not infringe privately owned rights. The views expressed in this paper are not necessarily those of the U.S. Government or the sponsoring agency. 


\section{VERIFICATION AND VALIDATION OF THE TRITIUM TRANSPORT CODE TMAP7}

\author{
Glen R. Longhurst \\ Idaho National Engineering and Environmental \\ Laboratory \\ P.O. Box 1625 \\ Idaho Falls, Idaho 83415-3860 \\ (208) 526-9950,gxl@inel.gov
}

The TMAP code has been upgraded to version 7 , which includes radioactive decay along with many features implemented in prior versions. Pursuant to acceptance and release for distribution, the code was exercised in a variety of problem types to demonstrate that it provides results in agreement with theoretical results for cases where those are available. It has also been used to model certain experimental results. In this paper, the capabilities of the TMAP7 code are

demonstrated by presenting some of the results from the verification and validation process.

\section{INTRODUCTION}

The TMAP Code was written at the Idaho National Engineering and Environmental Laboratory in the late 1980 s as a tool for safety analysis of systems involving tritium. Details of code theory and application are in Ref. [1] Its most recent upgrade to TMAP7 includes up to three separate traps, up to 10 diffusing species, and up to 30 surface-only species, which may be important in surface molecule formation but do not, themselves, diffuse in the material. Under solution-law dependent diffusion boundary conditions, such as Sieverts' law, TMAP7 automatically generates heteronuclear molecular partial pressures for functional enclosures when solubilities and partial pressures of the homonuclear molecular species are provided. In the new surfdep diffusive boundary condition mode, TMAP7 allows the user to include a surface binding energy, $E_{c}$, and an adsorption barrier energy, $E_{h}$. It includes asymmetrical diffusion between the surface sites and regular diffusion sites in the bulk. All of the previous features for heat transfer, flows between enclosures, and chemical reactions within the enclosures have been retained. One additional feature unique to TMAP7 is radioactive decay for both trapped and mobile species.

A prior version, TMAP2000, had many of these features. Some shortcomings with treatment of surface interactions were not previously resolved, so TMAP2000 was not distributed. Those shortcomings have now been overcome, and the code has been successfully demonstrated through an extensive verification and validation process [2]. Our objective in this paper is to highlight new

\author{
James Ambrosek \\ Department of Chemical and Fuels Engineering \\ University of Utah \\ Salt Lake City, Utah 84112 \\ (801) 581-3129
}

capabilities by comparing its performance with theoretical solutions and modeling some interesting experiments.

We present two groups of problems: (1) idealized problems for which analytic solutions are available, and (2) a simulation of an experiment.

\section{IDEALIZED PROBLEMS WITH THEORETICAL SOLUTIONS}

There exist numerous closed-form and series solutions for a variety of idealized approximations to real physical situations. Some of these include depleting of a reservoir through a diffusion membrane, heat and mass transfer through composite structures, chemical reactions in enclosures, mixing and equilibration of gases in a series of connecting enclosures, and simple radioactive decay. Three will be highlighted here to demonstrate code capabilities. The first is a pair of problems to demonstrate the effects of different kinds of traps. The second is the isotopic equilibration of two homonuclear species on a reactive surface. Finally, a radioactive decay problem in a distributed trap will be presented.

\section{II.A. Trapping}

Two distinctly different permeation transient responses are possible through a membrane that has traps in it, depending on the comparative strength and concentration of the traps. In one, referred to as the effective diffusivity regime, the transient is nearly the same shape as if there were no traps but the diffusivity is reduced to an effective value lower than would describe steady-state permeation. In the other, referred to as the strong trapping regime, there is effectively no permeation flux until the traps have been filled, then permeation turns on very suddenly. The demonstration of these modes is in a contrived material, which, for convenience, has a lattice number density of $3.16 \mathrm{E}+22$ atom $/ \mathrm{m}^{3}$, a thickness of $l=1 \mathrm{~m}$, and a constant temperature of $1,000 \mathrm{~K}$. Both problems begin with the membrane empty of gas atoms but the upstream surface held constant at a dissolved gas atom concentration $c_{o}=10^{-4}$ times the lattice density. A single trap was assumed in each case as was gas 
diffusivity, $D$, of unity. For the effective diffussivity solution, the trap concentration was taken to be $10 \%$ of lattice density, and the trapping energy was taken as $0.0086 \mathrm{eV}$. The theoretical transient history is given by

$$
J_{p}=\frac{c_{o} D}{l}\left[1+2 \sum_{m=1}^{\infty}(-1)^{m} \exp \left(-m^{2} \frac{\pi^{2} D_{e f f} t}{l^{2}}\right)\right]
$$

where

$$
D_{e f f}=\frac{D}{1+\frac{1}{\varsigma}}
$$

The trapping parameter, $\zeta$, is defined by

$$
\varsigma=\frac{\lambda^{2} v}{D_{o} \rho} \exp \left(\frac{E_{d}-\varepsilon}{k T}\right)+\frac{c}{\rho}
$$

Here, $\lambda$ is the lattice constant, $v$ is the Debye frequency, $D_{o}$ and $E_{d}$ are diffusivity pre-exponential and activation energy, respectively, $\varepsilon$ is the trapping energy, $k$ is Boltzmann's constant, $T$ is temperature, $c$ is the mobile atom concentration, and $\rho$ is trap concentration. Fig. 1 compares the permeation transient calculated by TMAP7 with the theoretical solution. They are indistinguishable.

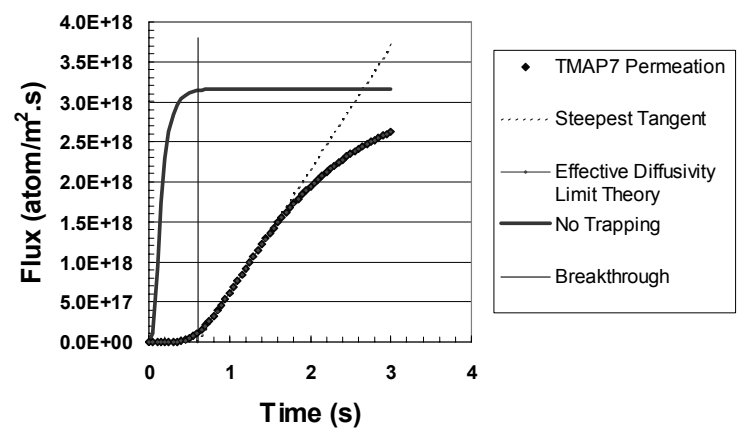

Fig. 1. Permeation transient in the effective diffusivity limit.

For the strong trapping limit, the trap energy was increased to $8.6 \mathrm{eV}$. Now, breakthrough comes at time

$$
\tau_{b_{d}}=\frac{l^{2} \rho}{2 c_{o} D}
$$

and steady-state permeation is then

$$
J_{p}=\frac{c_{o} D}{l}
$$

Fig. 2 shows the TMAP7 results and the theoretical expectation for breakthrough in that limit.

\section{II.B. Equilibration}

In an enclosure containing a mixture of $\mathrm{H}_{2}$ and $\mathrm{D}_{2}$ at time zero, if the surface supports quasi-instantaneous

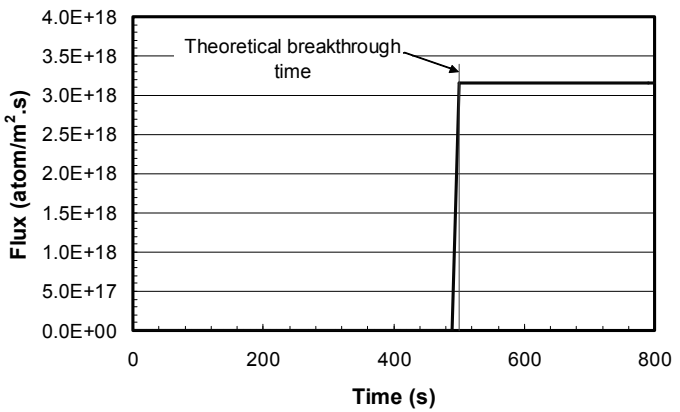

Fig 2. Permeation transient in the strong trapping limit.

isotopic exchange, the two homonuclear species will recombine to an equilibrium concentration of HD such that

$$
P_{H D}=\frac{2 P_{H_{2}}^{o} P_{D_{2}}^{o}}{P_{H_{2}}^{o}+P_{D_{2}}^{o}}\left[1-\exp \left(\frac{S}{V} \sqrt{\frac{k T}{2 \pi M}} t\right)\right]
$$

where $S$ is the surface area available for reaction, $V$ is the enclosure volume, $M$ is the average mass of the gas molecules, and other variables are as previously defined. If equilibration at the surface is not instantaneous but is rate-limited by dissociation, recombination, and/or desorption processes, then Equation (6) becomes

$$
P_{H D}=\frac{2 P_{H_{2}}^{o} P_{D_{2}}^{o}}{P_{H_{2}}^{o}+P_{D_{2}}^{o}}\left[1-\exp \left(\frac{S k T}{V} \frac{K_{d} K_{b}}{K_{r}+K_{b}} t\right)\right]
$$

where $K_{d}$ is the gas deposition rate per unit pressure

$$
K_{d}=\frac{1}{\sqrt{2 \pi M k T}} \exp \left(-\frac{E_{h}}{k T}\right)
$$

$K_{b}$ is the rate constant for molecular dissociation on the surface, and $K_{r}$ is the rate constant for desorption from the surface, given, respectively, by

$$
\begin{aligned}
K_{b} & =v \exp \left(-\frac{E_{b}}{k T}\right) \\
K_{d} & =v \exp \left(\frac{E_{c}-E_{h}}{k T}\right)
\end{aligned}
$$

For a demonstration problem, we selected a $1-\mathrm{m}^{3}$ enclosure with a 5-cm x 5-cm reactive surface area for equilibration. The volume initially held $10^{4} \mathrm{~Pa}$ of $\mathrm{H}_{2}$ and $10^{4} \mathrm{~Pa}$ of $\mathrm{D}_{2}$ but no HD. The temperatures of the enclosure and the surface were $1,000 \mathrm{~K}$. We chose $E_{h}-E_{c}$ $=0.06 \mathrm{eV}$, a Debye frequency of $8.4 \mathrm{E}+12 \mathrm{~s}^{-1}$, and the dissociation energy of zero for this calculation. Fig. 3 compares the TMAP7 calculation for growth history of $\mathrm{HD}$ in the enclosure with the theoretical predictions. A similar calculation for the same experiment configuration but with unequal starting pressures of $\mathrm{H}_{2}$ and $\mathrm{D}_{2}$ showed agreement with Equation (7) that was equally good. 


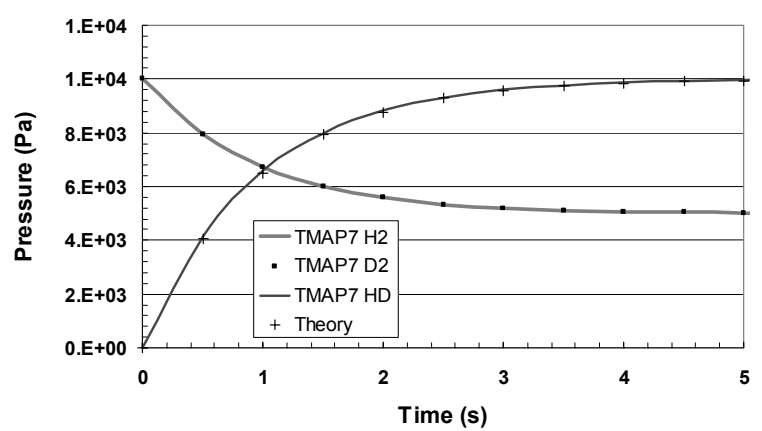

Fig. 3. Isotopic equilibration of $\mathrm{H}_{2}$ and $\mathrm{D}_{2}$ to form $\mathrm{HD}$.

\section{II.C. Radioactive Decay}

Fig. 4 shows the initial concentration of tritium in distributed traps in a slab $1.5 \mathrm{~m}$ thick. Trapping energy was $4.2 \mathrm{eV}$. After nearly 45 years of decay, all but $8 \%$ of the tritium had transmuted to He-3, exactly in line with theoretical results.

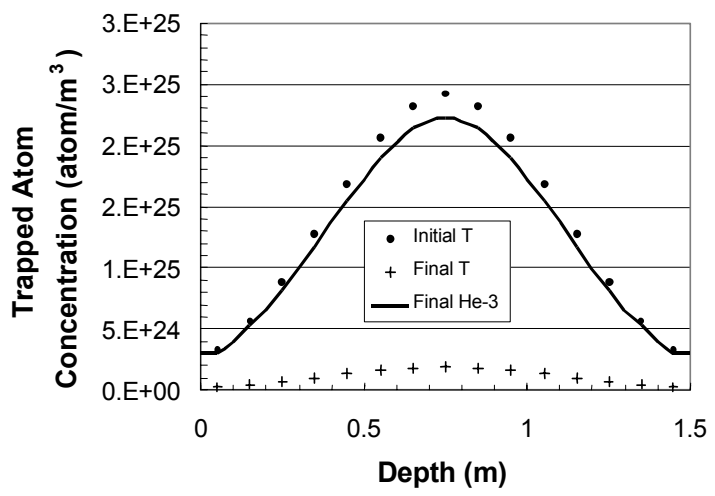

Fig. 4. Radioactive decay of tritium in distributed traps.

\section{SIMULATION OF EXPERIMENT}

To exercise the model, an experimental result of Hino et al. [3] was selected for approximation. In this experiment, $\mathrm{H}_{3}^{+}$was implanted at $5 \mathrm{keV}$ and a flux of $1 \times 10^{19} \mathrm{H} / \mathrm{m}^{2}$ s for 5,000 seconds into a polycrystalline tungsten foil $50 \times 50 \mathrm{~mm}^{2}$ and $0.1 \mathrm{~mm}$ thick at room temperature. Background pressure in the implantation chamber was $10^{-3} \mathrm{~Pa}$ while the implantation was going on and $10^{-5} \mathrm{~Pa}$ at other times. Following the implantation, the sample was subjected to thermal desorption spectroscopy by heating under vacuum at $50 \mathrm{~K} / \mathrm{min}$ to $1,273 \mathrm{~K}$ and then held at that temperature for several minutes.

We modeled this system with TMAP7 using the structure of Fig. 5. The implantation chamber (Encl 1) was assumed to have a volume of $0.1 \mathrm{~m}^{3}$ and to be evacuated by a $70 \mathrm{~L} / \mathrm{s}\left(0.07 \mathrm{~m}^{3} / \mathrm{s}\right)$ vacuum pump. It was defined for this problem as a functional enclosure having a preprogrammed temperature of $300 \mathrm{~K}$ for 5,000 seconds followed by a ramp to $1,273 \mathrm{~K}$ at a ramp rate of $50 \mathrm{~K} / \mathrm{min}$. The vacuum pump is represented by a boundary enclosure (Encl 2) held at $10^{-8} \mathrm{~Pa}$. Background pressure is supplied by Encl. 3 .

On the basis of TRIM [4] calculations, implantation was assumed to follow a normal distribution, peaking at $4.6 \mathrm{~nm}$ below the surface and having a scatter or characteristic half width of $3 \mathrm{~nm}$. Implantation was active for 5000 seconds and then was terminated.

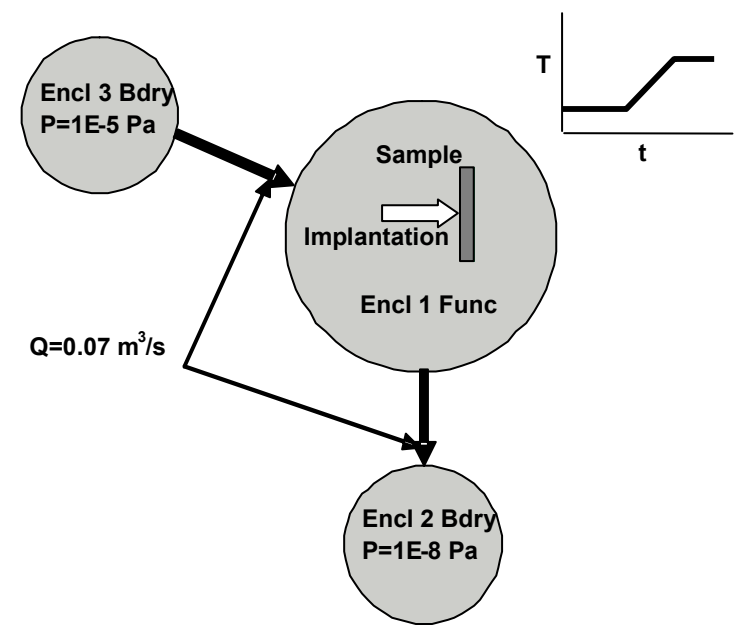

Fig. 5. Schematic of system used to model experiment of Hino et al. ${ }^{3}$

The diffusion boundary condition employed was Sieverts' law with Frauenfelder [3] solubility.

$$
S=1.83 \times 10^{24}\left(\frac{H}{m^{3}}\right) \exp \left(-\frac{1.04 \mathrm{eV}}{R T}\right)
$$

Diffusivity used for hydrogen through tungsten was the normally accepted Frauenfelder value [5].

$$
D=4.1 \times 10^{-7}\left(\frac{\mathrm{m}^{2}}{\mathrm{~s}}\right) \exp \left(-\frac{0.39 \mathrm{eV}}{R T}\right)
$$

Three traps were assumed in the sample. Trap concentrations and distributions were considered adjustable parameters while energies were determined by peak temperatures. The first was assumed to be associated with implantation (damage and precipitation) and to be normally distributed with a peak at $4.6 \mathrm{~nm}$ and a characteristic width of $10 \mathrm{~nm}$, consistent with the observations of Haasz et al. [6] that the damage zone exceeds the implantation depth. Its trap energy was adjusted, based on the temperature of the first peak, to be $1.3 \mathrm{eV}$, and it was assumed to be 0.1 atom fraction at the peak. The second was a uniform trap, probably associated with dislocations and was assigned a trap release energy of $1.7 \mathrm{eV}$, typical of but slightly higher than that seen by Anderl et al. [7] Its concentration was adjusted to $45 \mathrm{appm}$. The third trap was also assumed to be uniformly 
distributed and to have a trapping energy of $3.3 \mathrm{eV}$, a little greater than the deep trap seen by Frauenfleder [5] with a concentration of 15 appm.

These values gave a peak surface flux averaged over both sides of the sample of $10^{18} \mathrm{H}_{2} / \mathrm{m}^{2} \mathrm{~s}$ at 500 seconds into the desorption, to match the flux quoted by Hino et al. Because the experimental flux measurement was probably made from a pressure measurement having a background with a temporal drift, an estimated background pressure proportional to temperature is added to the calculated permeation flux data in Fig. 6.

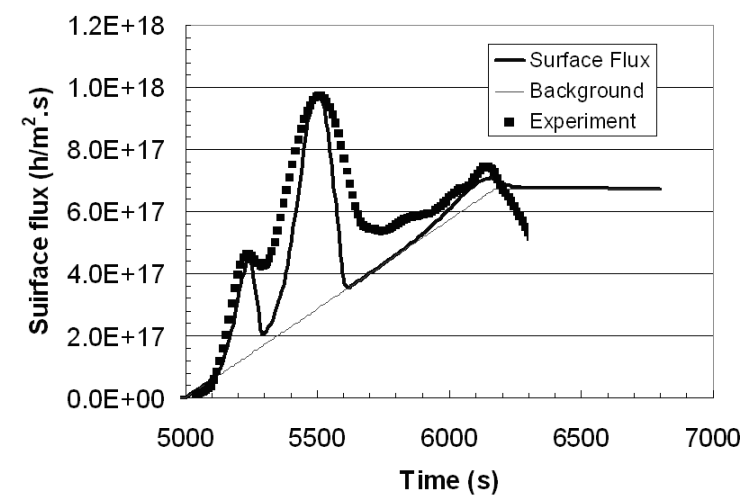

Fig. 6. Comparison of results from a representative problem computation (solid line) with experimental data from Hino et al. [3]. Total calculated result includes surface diffusive flux and temperaturedependent background signal.

The fit with the Hino et al. data is not exact because of several factors, the most prominent of which is probably the two-dimensionality of the experiment arising from beam non-uniformity and radial diffusion. Desorption of $\mathrm{H}^{2}$ from surrounding surfaces as the test article support structure was heated may also be important.

What we have modeled as evolution from the third trap may in reality be just a thermally induced background shift. It is also possible that it represents evolution from a trap type with a spectrum of trap energies, but determining that is beyond the present scope.

\section{CONCLUSION}

The TMAP code has been very useful over the years since its first development. It has been used in numerous safety calculations, experiment simulations, and design checks. Changes made to the TMAP code to convert it to TMAP7 should improve the functional usefulness of the code for a variety of applications where previous versions have been used. The elements of code utilization remain essentially the same, but added features of multiple traps, radioactive decay, and surface only species, with surface- energy dependent diffusion boundary conditions combined with some refinements in the way gas partial pressures are calculated in functional enclosures, have resulted in significant changes in the code's usefulness. It also no longer requires a separate FORTRAN compiler to run, making use of the GNU free license F77 compiler, which is distributed with the TMAP7 code.

\section{ACKNOWLDEGEMENT}

The authors are indebted to the many users of the earlier versions who have provided feedback on desirable added features and corrections needed to either the code or to documentation.

This work was performed for the U.S. Department of Energy, Office of Science under DOE-ID Operations Office Contract DE-AC07-99ID13727.

\section{REFERENCES}

[1]. G. R. Longhurst, TMAP7 User Manual, INEEL/EXT04-02353, Idaho National Engineering and Environmental Laboratory, Idaho Falls, Idaho (2004).

[2]. J. Ambrosek and G. R. Longhurst, Verification and Validation of TMAP7, INEEL/EXT-04-01657, Idaho National Engineering and Environmental Laboratory, Idaho Falls, Idaho (2004).

[3]. T. Hino et al., "Hydrogen retention properties of polycrystalline tungsten and helium irradiated tungsten," Fusion Engineering and Design, 39-40 (1998) 227-233.

[4]. J. F. Ziegler, J. P. Biersack, and U. Littmark, The Stopping and Range of Ions in Solids, Pergammon Press, New York (1985, new edition in 2003); see also internet site http://www.srim.org/.

[5]. R. Frauenfelder, "Solution and Diffusion of Hydrogen in Tungsten," Journal of Vacuum Science and Technology, 6 (1969) 388-397.

[6]. A. A. Haasz et al., "The effect of ion damage on deuterium trapping in tungsten," Journal of Nuclear Materials, 266-269 (1999) 520-525.

[7]. R. A. Anderl et al., "Hydrogen Transport and Trapping in Polycrystalline Tungsten," Fusion Technology, 21 (1992) 745-750. 